\title{
Esim Mechanism
}

\section{Sukhmeetsingh Guruwada, Prof. Vandana Navale}

Department of Computer Engineering, DPES Dhole Patil College of Engineering Pune, Maharashtra, India

Article Info

Volume 7, Issue 2

Page Number: 01-04

Publication Issue :

March-April-2021

Article History

Accepted : 01 March 2021

Published : 04 March 2021

\section{ABSTRACT}

With $5 \mathrm{G}$ technology physical separation of SIM cards with mobile phone will be possible in coming days. Physical separation will help connecting remotely to various devices and easily for the mobile users to switch to new handsets with all data available on the other device too. The embedded Universal Circuit Card is form of programmable SIM card that can be embedded directly into a device with an objective to avoid to avoid sim card change in case where data, the application type or the operator is changed with less cost, less time and more efficiency. Major concern with use of ESIM is the mutual security between the user and the operator.

Keywords : 5G, Esim, Embedded Systems, GSMA, EUICC

\section{INTRODUCTION}

SIM cards widely used in all mobile phones are used for securely storing the international mobile subscriber identity number (IMSI) and its related key. Its a harware chip and size have differ from large to tinest form in today's mobile devices. SIM cards are the one that will help identify and subscribe subscribers on mobile telephony devices. Physical process to activate sim cards for use is via document identification, telecom operators will activate IMSI number of the subscriber only after document verification. With emerging digitalization and tremendous gain in research for next generation technology, ESIM has emerged as an alternative option for physical chip of SIM cards. ESIM or embedded sim or embedded universal integrated circuit card or simply called as eUICC is a form of programmable SIM card that is embedded directly into a device. Embeding directly into smart devices will reduce the burden and costing for physical sim chips hence most of the $5^{\text {th }}$ generation devices have developed esim support for the devices.

\section{SIM Card}

A SIM card also known as subscriber identity module is globally used in smartphone devices. Its a smart card that stores the data for end users or subsribers of GSM phones. Global system for mobile communication regulates the process of user subscription for respective networks. SIM cards are small cards which contains a chip and are removable and transferable to other phones. Physically SIM card can be recognized as a tiny piece of plastic that has a small integrated chip which holds unique identification number, phone number and other required information that is related to the consumer.

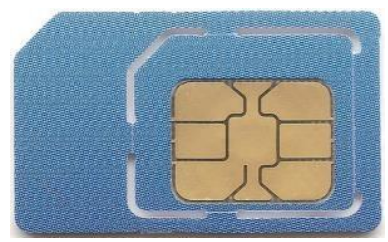

Fig 1. SIM Card 


\section{ESIM}

ESIM type of sim that will be directly embedded into the device replacing the physical chip as sim cards.

ESIM has been dubbed as next evolution of sim card, because it offers users the ability to change service provider over the air (OTA), without need to change physical embedded sim card itself. ESIM is basically a small programmable chip inside phone devices and works similar to NFC chips. ESIM information is rewritable, this means users can change the mobile operator with a simple phone call. Easy to add a data plan connecting devices with eSIMS to a mobile account can be done in few seconds. ESIM is regaulated by GSMA which has defined standards for ESIMs globally.

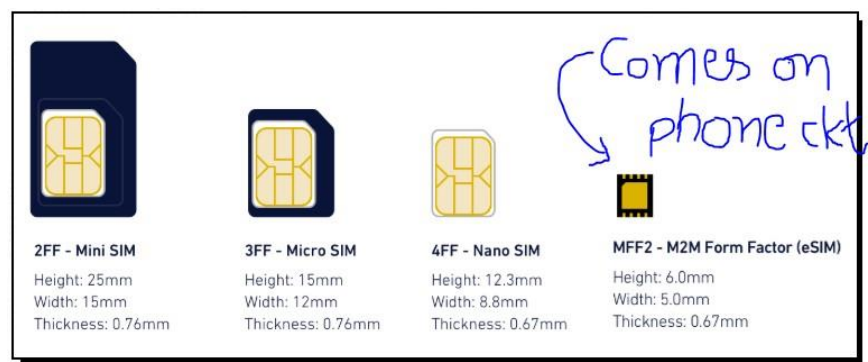

Fig 2. SIM to ESIM generation
eSIM extends the reach of the secure facilities from specific physical locations, to any location where the device can be reached over the internet. eSIM protocols provide security and integrity for data transfer. In sim cards distribution channel also contains business logic required by various service models. Logic may even represent about secured information like who has the control for the connections. Practically, its not possible to combine traditional build logic of SIM cards to eSIM hence, GSMA has created solutions suited for different type of channels as listed below.
A) M2M Solution

M2M solutions are also known as server-driven push models where server is the source. In server driven solution mobile device normally operates without any end user control of connectivity. It is managed by backend infrastructure, for example, biling, provisioning and CRM systems [1].

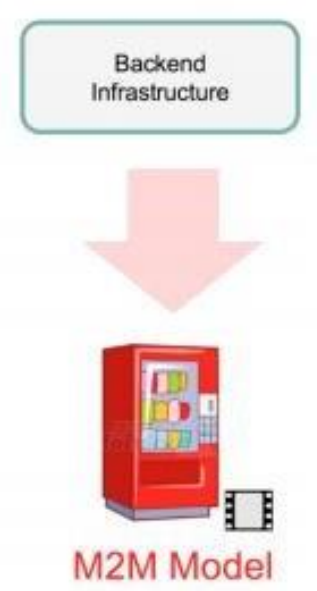

Fig 3. M2M Model

\section{B) Consumer Solution}

Consumer solution is known as a client driven pull model. Consumer solution as the name defines the end user that wants to use the eSIM will send an request for service thus initiating the communication from users end. Consumer solution manages end user interaction via the mobile device end user interface, and also supports standalone and companion device types.

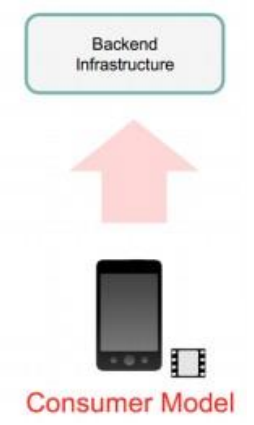

Fig 4. Consumer Model 


\section{Remote Provisioning Overview}

The GSMA Consumer solution has been developed from the base provided by the M2M solution, plus consideration of requirements for end user-managed devices [4].

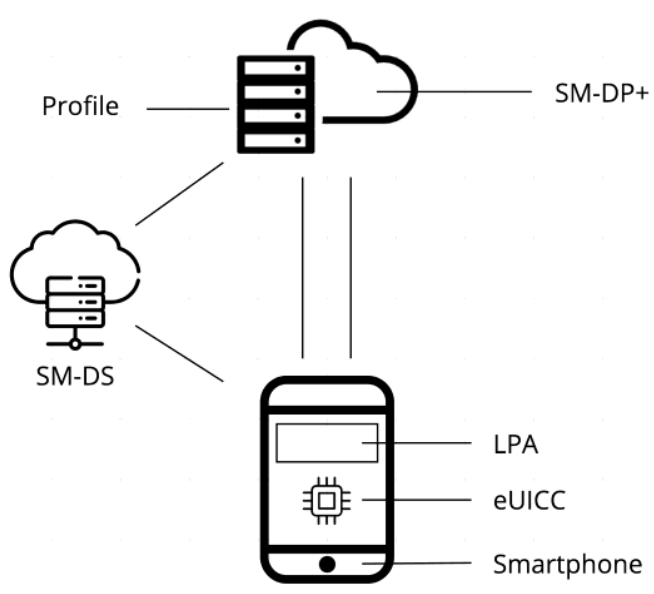

Fig 5. Remote provisioning

Let us understand the basic entities role included in remote provisioning management.

\section{- $\quad$ SM-DS}

SM-DS stands for Subscription Manager - Discovery Server that provides means for SM-DP+ to reach eUICC. The SM-DS functions as a go-between, allowing the SM-DP+ to locate the eUICC (device) without needing to ascertain what network the eUICC is connected to. Simply put, it acts as a secure notice board where the SM-DP+ can send a notification. The SM-DP+ then tells the LPA that it sent a notification and that a profile can be downloaded, and the internal LPA will then go receive that notification and commence the download of the profile. The purpose of an SM-DS is to hold a list of profiles that are available to an end user in a consumer eUICC environment.

\section{- $\quad \mathrm{SM}-\mathrm{DP}+$}

SM-DP also known as Subscription Management Data Preparation is responsible for various basic operations viz enabling, disabling, update and delete operations related to profile and also ensures a secure connection when a profile is sent to the eUICC.

\section{- $\quad$ Profile}

A profile is something that contains information or contract information from your service provider (carrier), such as the number assigned to your eSIM. For a physical SIM card, this is written into the SIM itself, while an eSIM can download this information from the network using OTA (over-the-air) technology. The eSIM profile is a virtual profile that stores the user's subscription and network settings and allows the user to connect to the corresponding mobile network.

- $\quad$ LPA

Local Profile Assistant is a set of functions that downloads encrypted profiles to the eUICC. It further acts as the local (device) user interface for the management of the downloaded profiles.

\section{Profile Activation}

How does the profile activation actually works out let us understand it with an basic example as referred in the figure below [3].

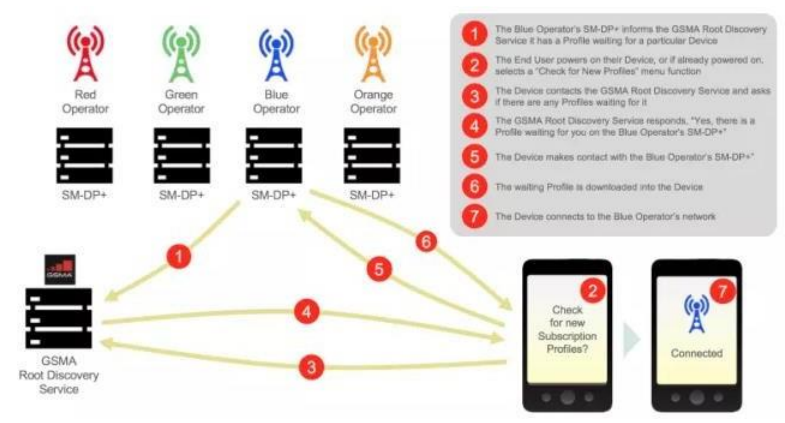

Fig 6. Profile Activation

1. A Blue operator's SM-DP+ informs the GSMA root discovery service it has a Profile waiting for particular mobile device

2. The End user powers on their device and selects a "Check for new Profiles" menu function. 
3. The device contacts GSMA root discovery service and asks if there are any Profile waiting for it.

4. The GSMA root discovery service responds. "Yes, there is a Profile waiting for you on Blue operator's SM-DP+".

5. The device makes contact with Blue operator's SM-DP+.

6. The waiting profile is downloaded into the device.

7. The device finally connects to Blue operator's network.

\section{CONCLUSION}

We discussed thorough change of SIM cards in the article focusing on basic details about ESIM and its profile activation entities to understand the end users flow with the current GSMA defined ESIM technology that has changed the physical chip to embedded chip directly into the devices. ESIM technology will help reduce physical chip cost and ease for changing operators on a single click go or just a call away. Globally soon next generation mobile phones will replace sim trays with esim integrated chips.

\section{REFERENCES}

[1]. GSMA, "Remote Provisioning Architecture for Embedded UICC Technical Specification"

[2]. M. Tsurusawa, "Latest Trends in Remote SIM Provisioning Technology", Special feature, The future of cellular networks, New Breeze Summer 2017

[3]. Esim White Paper, The what and how of Remote SIM Provisioning, March 2018

[4]. GSMA's https://www.gsma.com
Cite this article as :

Sukhmeetsingh Guruwada, "Esim Mechanism", International Journal of Scientific Research in Computer Science, Engineering and Information Technology (IJSRCSEIT), ISSN : 2456-3307, Volume 7 Issue 2, pp. 01-04, March-April 2021. Available at doi : https://doi.org/10.32628/CSEIT21721 